(c) SMOLIAROVA T.E.

UDC 537.9

DOI: $10.20333 / 2500136-2021-2-97-99$

\title{
Synthesis, properties and functionalization of gold nanostars for medical diagnostics
}

T. E. Smoliarova ${ }^{1,2}$

${ }^{1}$ Federal Research Center «Krasnoyarsk Science Center of the Siberian Branch of the Russian Academy of Sciences», Krasnoyarsk 660036, Russian

Federation

${ }^{2}$ Siberian Federal University, Krasnoyarsk 660041, Russian Federation

The aim of the research. To evaluate two approaches to colloidal synthesis using two different non-toxic surfactants (PVP and Triton X-100) and their properties for future use.

Material and methods. (PVP) The solution of GNSs with short tips was prepared in $200 \mathrm{ml}$ vial. $20 \mathrm{mg}$ of PVP (Polyvinylpyrrolidone, 1-ethenylpyrrolidin-2-one) were dissolved in the $200 \mathrm{~mL}$ of DMF (N,N-Dimethylmethanamide) (with the sonication to dissolve well). (Triton X-100) In a typical preparation of GNSs with long tips, the seed solution was prepared in a $20 \mathrm{~mL}$ vial: $5 \mathrm{~mL}$ of HAuCl4 $5 \cdot 10-4 \mathrm{M}$ in water are added to $5 \mathrm{~mL}$ of an aqueous solution of TritonX-100 0.1M. To examinate the shape and properties of prepared gold nanostars Cary 100 Bio Spectrophotometer using quartz cuvettes was used to taken on UV-Vis spectra. Transmission Electron Microscopy (TEM) was used to obtain shape and size of prepared GNSs.

Results. Microscopy analysis shows that the obtained GNSs have completely different shapes. The GNSs fabricated using synthesis approach with PVP have shorter tips and the cores are larger than the GNSs synthesized with Triton X-100 synthesis approach. TEM-images of the second ones show smaller size nanoparticles with the longer and thinner tips. Optical properties of the synthesized GNSs were analyzed using UV-vis-NIR absorbtion spectra, which shows maximum plasmon existence at $800 \mathrm{~nm}$ for GNSs synthesized with PVP and at $850 \mathrm{~nm}$ for GNSs synthesized with Triton X-100.

Conclusion. In summary, we developed GNSs using two colloidal synthesis approaches with the use of two different non-toxic surfactants (PVP and Triton $\mathrm{X}-100)$. In the future, gold nanostars are planned to be used to develop highly sensitive methods of medical diagnostics.

Key words: colloidal synthesis, gold nanostars, absorbtion spectra.

Conflict of interest. The authors declare the absence of obvious and potential conflicts of interest associated with the publication of this article.

Citation: Smoliarova TE. Synthesis, properties and functionalization of gold nanostars for medical diagnostics. Siberian Medical Review. 2021; (2):97-99. DOI: 10.20333/2500136-2021-2-97-99

\section{Introduction}

Gold nanoparticles exhibit strong localized surface plasmon resonances (LSPR) [1], which are associated with collective oscillations of conduction electrons that arise when the metal interacts with visible or near-infrared light. These resonances strongly depend on the shape, size and composition of particles, as well as on the dielectric properties of the metal itself and the environment [2]. Such customizability and sensitivity have stimulated the development of new synthetic strategies for controlling the shape of particles [3], which, in turn, allow us to anticipate the implementation of a number of potential applications in various fields, such as electronics [4], photonics. [5] or biosensors [6]. In some of these applications, in particular, in the so-called LSPR biosensor [7], the sensitivity of nanoparticles to changes in the local dielectric medium is an important parameter that should be taken into account. Since the sensitivity is determined by the degree of limitation of plasmon oscillations, which are responsible for the amplification of the near electric field at the particle surface, anisotropic metal nanoparticles such as rods, bipyramids, or stars have been identified as interesting systems. Gold nanostars (GNSs) are especially interesting because their intrinsic properties are the result of hybridization of plasmons concentrated in the core and tips of the nanoparticles. The core acts as an antenna that amplifies the electromagnetic field of the residual plasmons [8], and the morphology of the spikes (length or opening angle) and their number also have a strong influence on the frequency and intensity of plasmons. Here we report about two colloidal synthesis approaches with the use of two different non-toxic surfactants (PVP and Triton X-100) and their properties for future application.

\section{Material and methods}

(PVP) The solution of GNSs with short tips was prepared in $200 \mathrm{ml}$ vial. $20 \mathrm{mg}$ of PVP (Polyvinylpyrrolidone, 1-ethenylpyrrolidin-2-one) were dissolved in the $200 \mathrm{~mL}$ of DMF (N,N-Dimethylmethanamide) (with the sonication to dissolve well). Then the stirring plate with magnet was used for good dissolving of the following materials. $1093 \mu \mathrm{L}$ of the $\mathrm{HAuCl}_{4} 50 \mathrm{mM}$ were added to the solution and after $8 \mathrm{~min}$ the $150 \mu \mathrm{L}$ of the $15 \mathrm{~nm} \mathrm{Au}$ seeds were added to the stirring solution, after approximately 
10 min the color of the solution went from 'transparent' to dark blue. The solution was left stirring for $24 \mathrm{~h}$. And then the content was divided into 4 vials of $50 \mathrm{~mL}$ and washed.

(Triton X-100) In a typical preparation of GNSs with long tips, the seed solution was prepared in a $20 \mathrm{~mL}$ vial: $5 \mathrm{~mL}$ of $\mathrm{HAuCl}_{4} 5 \cdot 10^{-4} \mathrm{M}$ in water are added to $5 \mathrm{~mL}$ of an aqueous solution of TritonX-100 0.1M. The mixture is gently hand-shaken and a pale-yellow color is obtained. Then, $0.6 \mathrm{~mL}$ of a previously ice-cooled solution of $\mathrm{NaBH}_{4} 0.01 \mathrm{M}$ in water are added. The mixture is gently hand-shaken and a reddish-brown color appears. The seed solution is kept in ice and used in few hours (2 hours). The growth solution was prepared in a $20 \mathrm{~mL}$ vial, $250 \mu \mathrm{L}$ of $\mathrm{AgNO}_{3} 0.004 \mathrm{M}$ in water, $5 \mathrm{~mL}$ of $\mathrm{HAuCl}_{4} 0.001 \mathrm{M}$ in water are added in this order to $5 \mathrm{~mL}$ of an aqueous solution of TritonX-100 0.2M. Then, a 140-400 $\mu \mathrm{L}$ volume of an aqueous solution of ascorbic acid $0.0788 \mathrm{M}$ are added. The solution, after gentle mixing, becomes colorless. Soon after, $12 \mu \mathrm{L}$ of the seed solution are added. The solution is gently hand-shaken and a grey color appears and quickly changes to green and becomes more intense. The sample is allowed to equilibrate for $1 \mathrm{~h}$ at room temperature and then washed.

All the glassware used for seedgrowth methods was always pretreated before use. It was washed in aqua regia for 24 hours to remove Au seeds, then washed and filled with bidistilled water.

To examinate the shape and properties of prepared gold nanostars Cary 100 Bio Spectrophotometer using quartz cuvettes was used to taken on UV-Vis spectra. Centrifugation was carried out using the Universal 320 centrifuge (Hettich Zentrifugen) and Sorvall Legend
Micro 21R centrifuge with the use of polypropylene $50 \mathrm{~mL}$ tubes. The centrifugation speed was in the 5000-4500 rpm range.

Transmission Electron Microscopy (TEM) was used to obtain shape and size of prepared GNSs. Solutions of GNSs $(10 \mu \mathrm{L}$ ) were deposited on copper grids (400 mesh) covered with a carbon film. Images were taken using TEM Jeol JEM 2010.

\section{Results}

Microscopy analysis shows that the obtained GNSs have completely different shapes. The GNSs fabricated using synthesis approach with PVP have shorter tips and the cores are larger (Fig. 1(A)) than the GNSs synthesized with Triton X-100 synthesis approach. TEM-images of the second ones show smaller size nanoparticles with the longer and thinner tips (Fig. 1(B)).

Optical properties of the synthesized GNSs were analyzed using UV-vis-NIR absorbtion spectra (Fig. 2), which shows maximum plasmon existence at $800 \mathrm{~nm}$ for GNSs synthesized with PVP and at $850 \mathrm{~nm}$ for GNSs synthesized with Triton X-100.

\section{Conclusion}

In summary, we developed GNSs using two colloidal synthesis approaches with the use of two different non-toxic surfactants (PVP and Triton X-100). The results of the synthesis show that the have optical properties of synthesized nanoparticles are suitable for development of LSPR-biosensors and medicine applications such as be used as a photothermal material for the hyperthermic destruction of cancer using NIR light. In the future, gold nanostars are planned to be used to develop highly sensitive methods of medical diagnostics.
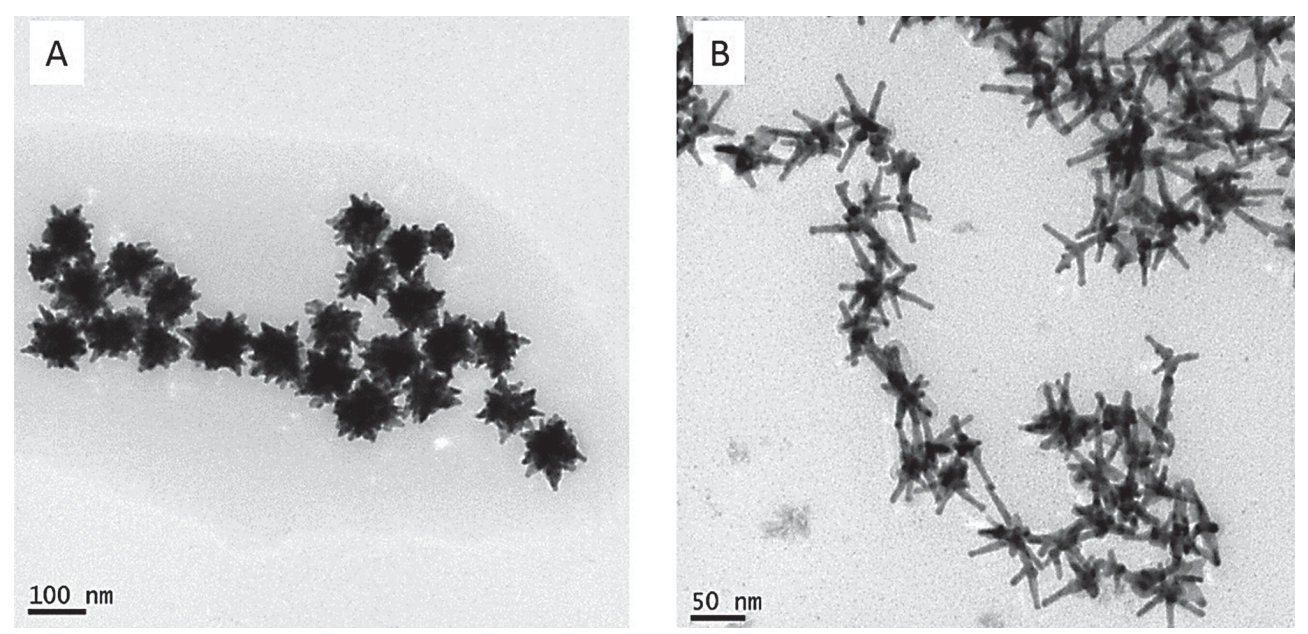

Figure 1. TEM-images of GNSs synthesized with (A) synthesis approach with PVP, (B) synthesis approach with Triton $X-100$. 


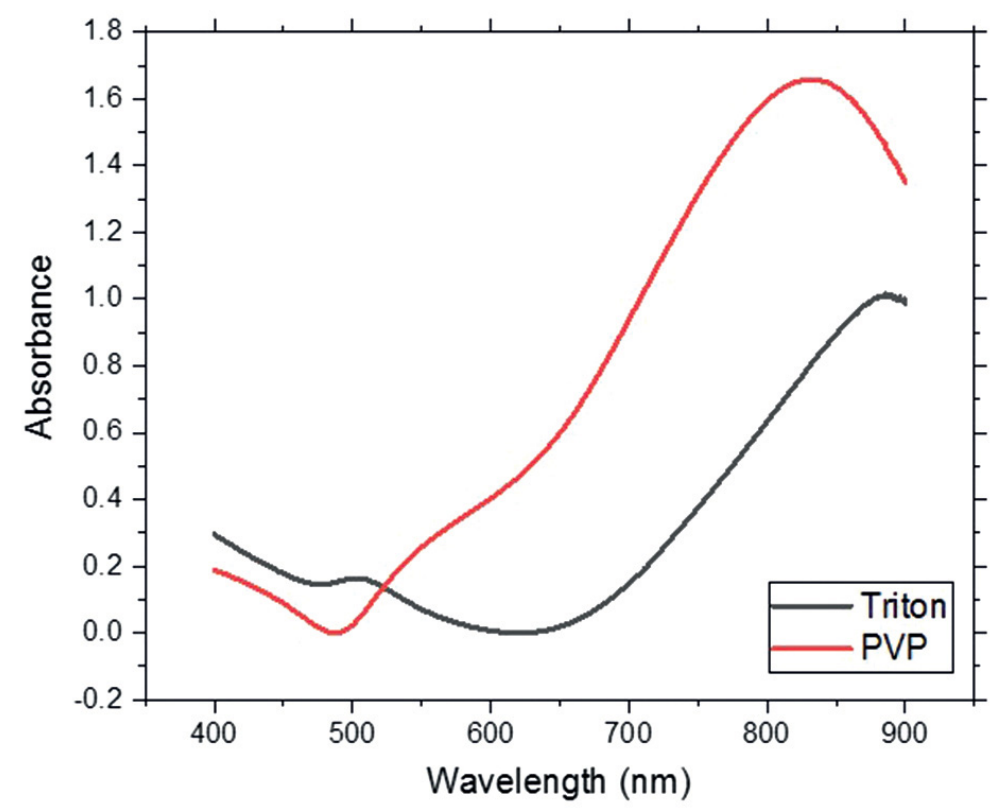

Figure 2. UV-vis-NIR absorption spectra obtained for GNSs synthesized with PVP and Triton X-100 surfactants.

\section{References}

1. Burda C, El-Sayed M. Chemistry and properties of nanocrystals of different shapes. Chemical Reviews. 2005; 105(4):1025-1102.

2. Kelly KL, Coronado E, Zhao LL, Schatz GC. The Optical Properties of Metal Nanoparticles: The Influence of Size, Shape, and Dielectric Environment. The Journal of Chemical Physics B. 2003; 107(3):668-677.

3. Pérez-Juste J, Pastoriza-Santos I, Liz-Marzán LM, Mulvaney P. Gold nanorods: synthesis, characterization and applications. Coordination Chemistry Reviews. 2005; (249):1870-1901.

4. Huang Yu, Duan X, Wei Q, Lieber CM. Directed assembly of one-dimensional nanostructures into functional networks. Science. 2001; 291(5504):630-633.

5. Matt L, Sirbuly DJ, Johnson JC, Goldberger J, Saykally RJ, Yang P. Nanoribbon waveguides for subwavelength photonics integration. Science. 2004; 305(5688): 1269-1273.
6. Katz E, Willner I. Integrated nanoparticle-biomolecule hybrid systems: synthesis, properties, and applications. Angewandte Chemie International Edition. 2004; 43(45): 6042-6108.

7. Munish C, Liz-Marzán LM. Coating matters: the influence of coating materials on the optical properties of gold nanoparticles. Nanophotonics. 2012; 1(3-4): 199-220.

8. Kumar M, Pethuraja GG, Singh VN, Mehta BR, Singh JP. Nanoparticle formation by swift heavy ion irradiation of indium oxide thin film. Nanotechnology. 2008; 19(17): 175606.

\section{Author information}

Tatiana E. Smoliarova, junior researcher, Federal Research Center "Krasnoyarsk Science Center of the Siberian Branch of the Russian Academy of Sciences"; Address: 50, Academgorodok st., Krasnoyarsk, Russian Federation 660036; Phone: +7(913)5693045; e-mail: smol.nano@ yandex.ru, https://orcid.org/0000-0002-0123-1965

Received 16 February 2021 Revision Received 18 March 2021 Accepted 31 March 2021 